\title{
Development of transgenic rice (Oryza sativa L.) expressing wheat high- and low-molecular-weight glutenin subunit proteins
}

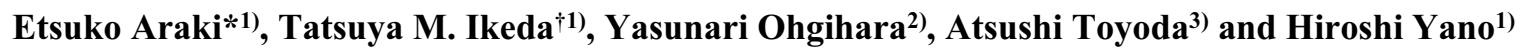 \\ 1) National Agricultural Research Center for Western Region, 6-12-1 Nishifukatsu, Fukuyama, Hiroshima 721-8514, Japan \\ 2) Kihara Institute for Biological Research of Yokohama City University, 641-12 Maioka, Totsuka, Yokohama, Kanagawa 244-0813, \\ Japan \\ 3) RIKEN Genomic Sciences Center, 1-7-22 Suehiro, Tsurumi, Yokohama, Kanagawa 230-0045, Japan
}

\begin{abstract}
We isolated transformation-competent artificial chromosome (TAC) clones harboring a high-molecularweight glutenin subunit (HMW-GS) gene and a low-molecular-weight GS (LMW-GS) gene from wheat genomic DNA, and developed transgenic rice lines with these genes using a transformation system mediated by Agrobacterium tumefaciens. We developed two lines of transgenic rice, one expressing the gene coding for HMW-GS and the other expressing the gene coding for LMW-GS. By crossing these transgenic lines, a novel line harboring both genes was developed and the expression of GS proteins was analyzed. This is the first study indicating that the two kinds of wheat GSs, HMW-GS and LMW-GS, accumulated in rice endosperm. In all the transgenic lines, the introduced GS genes were expressed in the rice endosperm, and the expressed proteins were processed at the same site as the mature GS protein in wheat seeds, forming insoluble polymeric proteins similar to those found in wheat. It was suggested that the protein-processing system was conserved between rice and wheat, and that it was possible to produce wheat gluten using the proteinprocessing system of rice.
\end{abstract}

Key Words: rice, wheat, high-molecular-weight glutenin subunit, low-molecular-weight glutenin subunit, transformation-competent artificial chromosome (TAC) vector.

\section{Introduction}

Storage proteins of rice (Oryza sativa L.) seeds are composed of albumins (water-soluble), globulins (salinesoluble), prolamins (alcohol-soluble) and glutelins (residue). These proteins accumulate in special vacuoles referred to as protein bodies. Two kinds of protein bodies occur in rice: endoplasmic reticulum (ER)-derived protein body-I (PB-I) and vacuolar protein body-II (PB-II) (Okita and Rogers 1996, Tanaka et al. 1980). PB-I, containing prolamins, accounts for approximately $20 \%$ of milled rice proteins, and PB-II, containing glutelins and globulins, for approximately $60 \%$ of milled rice proteins (Ogawa et al. 1987, Yamagata et $a l .1982$ ). Many storage protein mutants have been screened from seeds treated with chemical mutagens. Iida et al. (1993) produced a mutant line, LGC1, in which several glutelins, controlled by a single dominant gene $L g c l$, were severely suppressed. However, the levels of prolamin and globulin increased in compensation, and the total amount of seed protein did not differ from that in normal rice (Iida et al. 1997).

Communicated by M. Ohshima

Received August 23, 2007. Accepted January 22, 2008.

*Corresponding author (e-mail: earaki@affrc.go.jp)

$\uparrow$ Contribution equals to corresponding author
In wheat (Triticum aestivum L.), glutenins and gliadins are the major seed storage proteins which form a continuous proteinaceous matrix surrounding the starch granules (Parker 1982). The gluten network, which shows viscoelastic properties during dough mixing, is derived from this matrix and these properties are required for the processing of a wide variety of foods, such as breads, noodles and cakes. Among the gluten proteins, the glutenins consist of subunits with high (95 to $136 \mathrm{kDa}$ ) and low (36 to $44 \mathrm{kDa}$ ) molecular weight. The high-molecular-weight glutenin subunits (HMW-GSs) are encoded by the Glu-A1, Glu-B1 and GluD1 genes on the long arm of chromosomes 1A, 1B and 1D, respectively (Payne et al. 1980). The HMW-GSs were classified into $\mathrm{x}$-type and y-type based on the amino acid sequence. The low-molecular-weight glutenin subunits (LMW-GSs) are encoded by the Glu-A3, Glu-B3 and Glu$D 3$ genes on the short arm of these chromosomes (Gupta and Shepherd 1990, Jackson et al. 1983). If gluten proteins could be expressed in rice seeds, a gluten network could be formed from rice flour, and this flour might be used for the processing of various food products.

In the present study, we first screened clones harboring the GS genes from a wheat genomic library constructed with a transformation-competent artificial chromosome (TAC) vector (Liu et al. 2000) containing a large, approximately 40 kbp genomic DNA fragment, and analyzed the sequences of 
the inserted region. Then, we introduced the genes into LGC1 from selected TAC clones using a transformation system mediated by Agrobacterium tumefaciens. Since the GSs are water-insoluble proteins belonging to the prolamin superfamily (Shewry et al. 1995), in transgenic rice, they may accumulate within PB-I containing prolamins. We analyzed the expression of the genes in the transgenic lines by 2D gel electrophoresis as well as the N-terminal amino acid sequences of the GS proteins.

\section{Materials and Methods}

\section{Selection of wheat TAC clones with glutenin subunit}

A wheat genomic library constructed with the TAC vector (pYLTAC17; Liu et al. 2000) was used for the cloning of GS genes and transformation. A clone for the HMWGS gene was selected by PCR using the primers HMW5-1 (TCACTGGCTGGCCGACAATG) and HMW5-2 (GAAG GTGAGGCCTCTGAGCA) which were designed from the HMW-GS gene sequence on GenBank (accession no. X12928). A clone for the LMW-GS gene was selected by PCR using the primers Glu-4.2 (GCAACTTTGATGAT GAATCC) and Glu-5.4 (AAACAAAGGTGATCCAAC TAT) (Ikeda et al. 2006). PCR was performed in a total reaction solution of $25 \mu \mathrm{l}$ containing $1.5 \mathrm{mM}$ of $\mathrm{MgCl}_{2}$, $0.1 \mathrm{mM}$ of each dNTP, 5 pmol of each primer, $0.5 \mathrm{U}$ of AmpliTaq Gold DNA polymerase (Applied Biosystems, USA), PCR Gold buffer (Applied Biosystems) and $50 \mathrm{ng}$ of total DNA. The reaction was performed according to the following protocol using the GeneAmp PCR system 9700 (Applied Biosystems): denaturation at $94^{\circ} \mathrm{C}$ for $5 \mathrm{~min} ; 35$ cycles of $94^{\circ} \mathrm{C}$ for $30 \mathrm{~s}, 55^{\circ} \mathrm{C}$ for $30 \mathrm{~s}, 72^{\circ} \mathrm{C}$ for $1 \mathrm{~min}$; and a final extension at $72^{\circ} \mathrm{C}$ for $7 \mathrm{~min}$. PCR products were electrophoresed in $1 \%$ agarose gel in a TAE buffer.

\section{Analysis of selected TAC clones}

The copy number of the GS genes in the selected clones was estimated by Southern blot analysis using HMW-GS and LMW-GS gene-specific probes. TAC clones were digested with four restriction enzymes, BamHI, EcoRI, HindIII and $X b a I$. Southern hybridization was conducted using the ECL system (Amersham Biosciences, USA). The loci of the selected individual GS genes were determined using nullisomic-tetrasomic lines of Chinese Spring, CS-N1AT1B, CS-N1BT1D and CS-N1DT1B. PCR was performed using the GS-specific primers HMW 8-1.1 (CTAAGTGCCGTC TCGTC) and HMW 8-2.3 (TGCAGAGAATCTGGGTA GTG) which were designed from the HMW-GS gene sequence on GenBank (accession no. X12928) for the HMWGS gene, and Glu 24 (GCCGTTGCGCAAATTTC) and Glu 13 (TTGGGGCTGTTGTTGCTGATA) which were designed from the LMW-GS gene sequence on GenBank (accession no. U86030, Ikeda et al. 2002) for the LMW-GS gene, using genomic DNA of these nullisomic-tetrasomic lines. The reactions were performed as described above. The sequence of insert DNA was determined using BigDye ter- minator v 3.1 (Applied Biosystems).

\section{Plant materials and transformation}

Calli were induced from the embryos of mature seeds of LGC1 according to the method of Fukuoka et al. (2000). Genes for HMW-GS and LMW-GS were introduced into the calli derived from LGC1 using the Agrobacterium strain EHA101 with the selected TAC clones, 3-113 for HMW-GS and 4-228 for LMW-GS. Six $\mathrm{T}_{0}$ plants with the HMW-GS gene and four $\mathrm{T}_{0}$ plants with the LMW-GS gene were grown in a greenhouse and mature seeds were obtained individually from each plant. Progenies in the homozygous state for the HMW-GS gene or the LMW-GS gene were repeatedly selected by PCR and 2D-PAGE in each generation until the $\mathrm{T}_{5}$ generation.

Since there were no differences in the growth vigor among the homozygous plants expressing HMW-GS or LMW-GS, each of the $\mathrm{T}_{4}$ plants, in which the expression of HMW-GS or LMW-GS was confirmed, was designated as HMW-113 and LMW-228, respectively, and used in this analysis. To develop a rice line with both HMW-GS and LMW-GS genes, these lines were artificially crossed in the greenhouse. Then, the $\mathrm{F}_{2}$ population was grown in the greenhouse and homozygote lines with both HMW-GS and LMW-GS genes were selected from the segregating $\mathrm{F}_{3}$ lines using the PCR method described above.

\section{D-PAGE and $N$-terminal amino acid sequence analysis}

Total storage proteins were extracted from rice seeds using $50 \mathrm{mM}$ of Tris- $\mathrm{HCl}(\mathrm{pH} 8.0)$ containing $2 \%$ of SDS and $50 \%$ of 1-propanol in the presence of the reducing agent dithiothreitol (1\% DTT). For the extraction of the glutenin fraction, milled seeds were washed using $50 \%$ of 1 propanol. The glutenin fraction was extracted using $50 \mathrm{mM}$ of Tris- $\mathrm{HCl}(\mathrm{pH} 8.0)$ containing $50 \%$ of 1-propanol and 1\% of DTT. Each fraction was precipitated with $80 \%$ of acetone, and the resulting pellets were dissolved in $250 \mu \mathrm{l}$ of IEF sample buffer containing 16\% of isopropanol, $8.5 \mathrm{M}$ of urea, $4 \%$ of CHAPS, $25 \mathrm{mM}$ of DTT, and $0.5 \%$ of IEF buffer, $\mathrm{pH}$ 6-11 (Amersham Biosciences), according to the method of Ikeda et al. (2005). After mixing for $30 \mathrm{~min}$ at room temperature, the samples were applied to Immobiline Dry-Strip, $\mathrm{pH}$ 6-11 (Amersharm Biosciences), and the 13-cm strip was cut on the acidic side to $11 \mathrm{~cm}$ to fit the Protean IEF Cell (BioRad Laboratories, USA). After the samples were allowed to stand overnight in-gel rehydration, IEF was performed for a total of $18 \mathrm{kVh}$. The strip gels were equilibrated with $6 \mathrm{M}$ of urea, $2 \%$ of SDS, $20 \%$ of glycerol, $10 \mathrm{mM}$ of DTT, and 50 $\mathrm{mM}$ of Tris- $\mathrm{HCl}\left(\mathrm{pH} \mathrm{6.8)}\right.$, and stored at $-25^{\circ} \mathrm{C}$. For the second dimension, SDS-PAGE was performed using a $14 \%$ gel $(14 \mathrm{~cm} \times 14 \mathrm{~cm} \times 1 \mathrm{~mm}$, acrylamide : bis-acrylamide $=$ $29.6: 0.4)$ at $30 \mathrm{~mA}$ for $2.5 \mathrm{~h}$. The gels were stained with Coomassie Brilliant Blue G-250, according to the method of Neuhoff et al. (1988). To analyze the N-terminal amino acid sequence, the gels were blotted onto a Sequi-Blot PVDF Membrane (Bio-Rad Laboratories) with a Trans-Blot SD 
Semi-Dry Transfer Cell (Bio-Rad Laboratories). The Nterminal amino acid sequences of individual spots were determined with a PPSQ-21A protein sequencer (Shimadzu, Japan).

\section{Results}

Selection of wheat TAC clones with glutenin subunits and their characterization

From the TAC library constructed by Liu et al. (2000), we selected two TAC clones by PCR using GSs-specific primers. Clone 3-113 was selected using the HMW-GS gene-specific primer sets and clone 4-228 was selected using the LMW-GS gene-specific primer sets. The complete sequences of clones 3-113 and 4-228 were determined, and the restriction enzyme sites of BamHI, EcoRI, HindIII and XbaI were identified in the sequences of each clone. The insert sizes of clones $3-113$ and 4-228 were 53,888 and 46,433 bp, respectively. The copy number of the GS gene in each TAC clone was confirmed by Southern analysis with probes specific for GSs. In 3-113, a single band was detected in DNA digested with BamHI, EcoRI and $X b a \mathrm{I}$, and two bands were found in cloned DNA digested with HindIII (Fig. 1A). Since the sequence of HMW-GS included the HindIII and BamHI restriction enzyme sites (Fig. 2A), two bands found in DNA digested with HindIII were derived from one HMW-GS gene. Based on the sequence of 3-113, the DNA including HMW-GS was divided into two fragments, 2.9 and $2.5 \mathrm{kbp}$ long, using BamHI. These two fragments may not have been distinguishable in Southern analysis, due to their similar
$3-113$

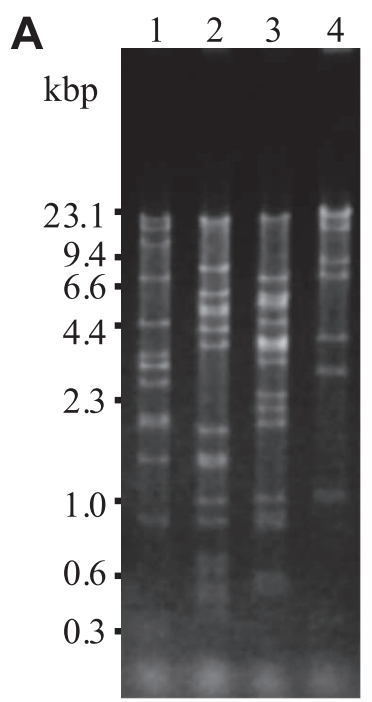

$4-228$

B

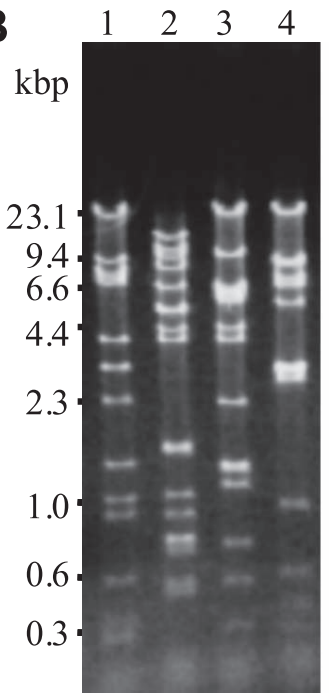

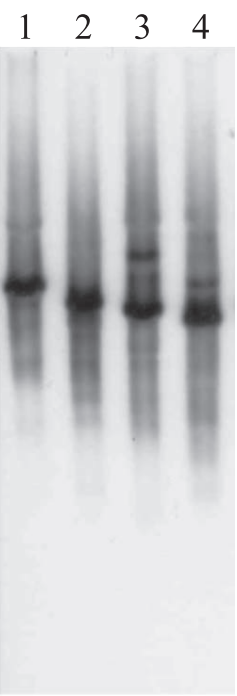

Fig. 1. Southern blot analysis of TAC clones with probes specific for the HMW-GS (A) and LMW-GS (B) genes. TAC clones were digested with the following restriction enzymes: 1, BamHI; 2, EcoRI; 3, HindIII; 4, XbaI.

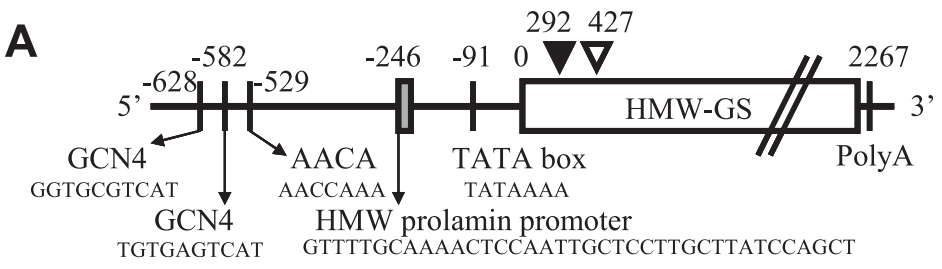

B

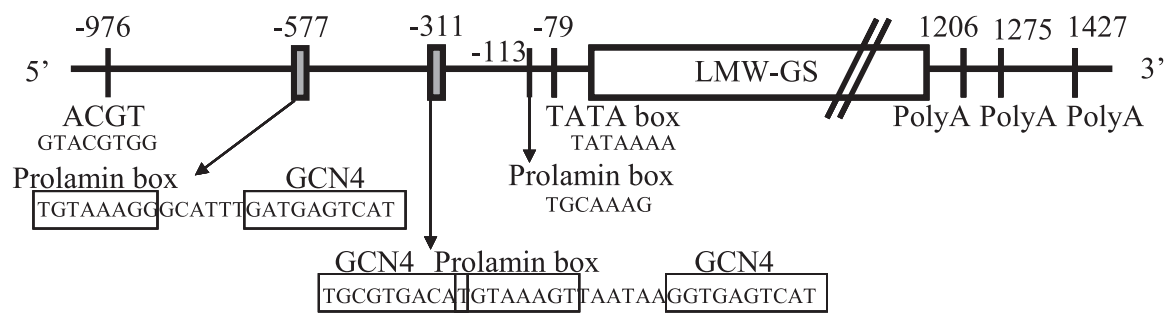

Fig. 2. Structure of the regulatory region of the TAC clone 3-113 harboring the HMW-GS gene (A) and of the TAC clone 4-228 harboring the LMW-GS gene (B). Restriction enzyme sites are indicated by black (BamHI) and white (HindIII) arrowheads. 
size. These results indicated that clone 3-113 contained a single copy of the HMW-GS gene. In clone 4-228, a single band was detected in the cloned DNA digested with BamHI and $E c o$ RI, and a major band and a minor band were found in the DNA digested with $X b a \mathrm{I}$ and HindIII (Fig. 1B). Since none of these restriction enzyme sites were included in the sequence of the LMW-GS gene (Fig. 2B), it was considered that the minor band was not derived from the LMW-GS gene. These results indicated that clone 4-228 also contained a single copy of the LMW-GS gene.

The deduced N-terminal amino sequences of these GSs are shown in Fig. 3. The expected amino acid sequence of HMW-GS in clone 3-113 was the same as that of B1y9 (Fig. 3A). In the PCR analysis using the nullisomictetrasomic lines of Chinese Spring with the HMW-GS genespecific primer (HMW 8-1.1/HMW 8-2.3), the PCR products were not detected in N1BT1D (Fig. 4A), indicating that the HMW-GS gene-specific primer could detect the gene on chromosome 1B of Chinese Spring. The expected amino acid sequence of LMW-GS in clone 4-228 showed a high similarity to that of Group 11 or Group 12 in the classification of Ikeda et al. (2002) (Fig. 3B). In the PCR analysis using the nullisomic-tetrasomic lines of Chinese Spring with the LMW-GS gene-specific primer (Glu 24/Glu 13), the PCR products were not detected in N1AT1B (Fig. 4B), indicating that the LMW-GS gene-specific primer could detect the gene on chromosome $1 \mathrm{~A}$ of Chinese Spring.

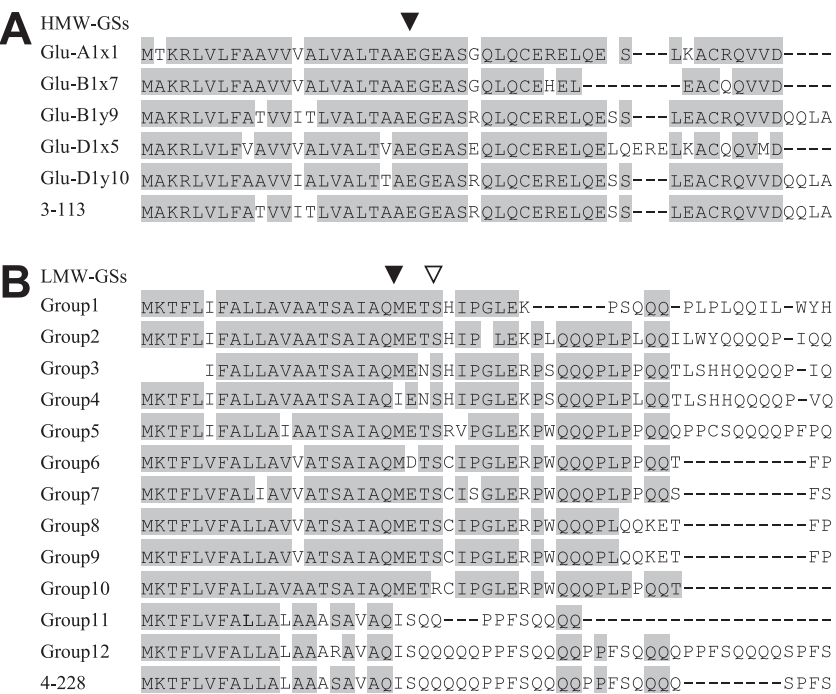

Fig. 3. Comparison of deduced N-terminal amino acid sequences of HMW-GSs (A) and LMW-GSs (B). Processing sites are indicated by black and white (Group3 and Group4) arrowheads. GenBank accession numbers are as follows: Glu-A1x1 (CAA43331), Glu-B1x7 (AAZ23584), Glu-B1y9 (S18733), Glu-D1x5 (CAA31395), Glu-D1y10 (P10387), Group1 (BAB78737), Group2 (BAB78738), Group3 (BAB78739), Group4 (BAB78750), Group5 (BAB78751), Group6 (BAB78754), Group7 (BAB78758), Group8 (BAB78759), Group9 (BAB78760), Group10 (BAB78761), Group11 (BAB78762), Group12 (BAB78763).
The structure of the flanking region of the HMW-GS gene is shown in Fig. 2A. In the $5^{\prime}$ upstream region of HMW-GS, a HMW prolamin promoter (Thomas and Flavell 1990) (-246 to -209) and a TATA box (-91 to -85) were identified. In the $3^{\prime}$ downstream region of HMW-GS, a polyA signal (2267 to 2273) was found as the terminator. Other sequences detected in the $5^{\prime}$ upstream region of HMW-GS consisted of two GCN4 boxes (Takaiwa et al. 1996) (-628 to -619 and -582 to -573 ) and an AACA motif (Takaiwa et al. 1996) (-529 to -524). The structure of the flanking region of the LMW-GS gene is shown in Fig. 2B. In the $5^{\prime}$ upstream region of LMW-GS, LMW prolamin boxes (Wu et al. 2000) $(-577$ to $-570,-301$ to -294 and -113 to -107$)$ and a TATA box (-79 to -73$)$ were identified. In the $3^{\prime}$ downstream region of LMW-GS, polyA signals (1206 to 1211,1275 to 1281 and 1427 to 1435 ) were found as the terminator. Other sequences observed in the $5^{\prime}$ upstream region of LMW-GS included an ACGT motif (Schmidt et al. 1992) (-976 to -969) and three GCN4 boxes (Takaiwa et al. 1996) (-563 to $-554,-311$ to -302 and -288 to -279$)$.

\section{Development of transgenic rice harboring wheat $H M W$ - and LMW-GS genes}

To select plants harboring either the HMW-GS gene or the LMW-GS gene in the homozygous state, progenies were repeatedly selected by PCR using specific primer sets and 2D-PAGE in each generation until the $\mathrm{T}_{5}$ generation. The PCR products in the transgenic lines $\left(\mathrm{T}_{5}\right.$ generation) are shown in Fig. 5. LGC1 did not harbor the HMW-GS and LMW-GS genes (lanes 7 and 8), whereas the transgenic rice HMW-113 with clone 3-113 exhibited a HMW-GS genespecific PCR product (lanes 5 and 6). The transgenic rice LMW-228 with clone 4-228 showed a LMW-GS genespecific PCR product (lanes 3 and 4).

To develop rice plants harboring both HMW-GS and LMW-GS genes, $F_{1}$ plants were obtained from a cross between the HMW-113 $\left(\mathrm{T}_{5}\right)$ and LMW-228 $\left(\mathrm{T}_{5}\right)$ lines. PCR
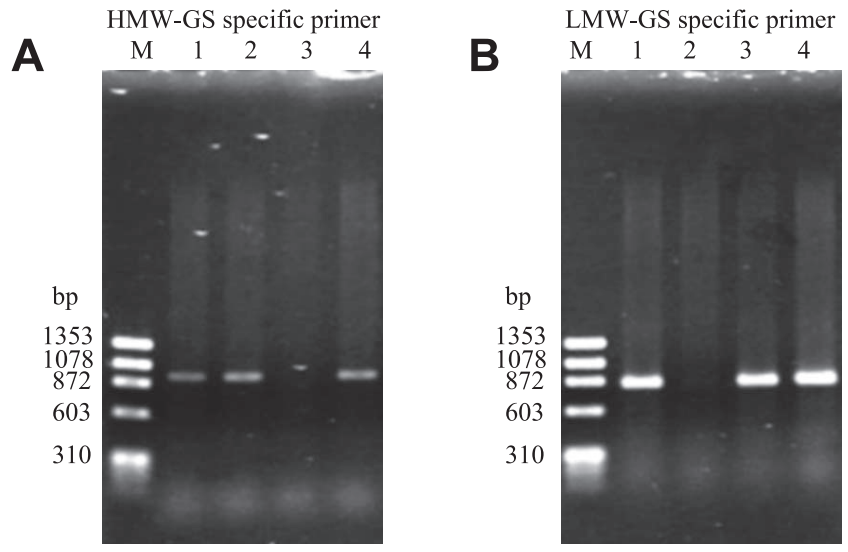

Fig. 4. PCR products in nullisomic-tetrasomic lines of Chinese Spring (CS) using primers specific for the HMW-GS (A) and LMWGS (B) genes. 1, CS; 2, CS-N1AT1B; 3, CS-N1BT1D; 4, CSN1DT1B. 


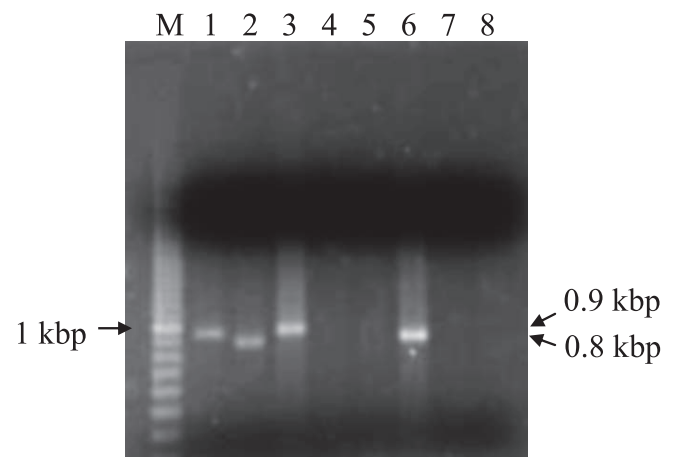

Fig. 5. HMW-GS and the LMW-GS genes in transgenic rice. Lanes 1 and 2, HLMW-GS; lanes 3 and 4, LMW-228; lanes 5 and 6, HMW-113; lanes 7 and 8, LGC1. The gene product of $0.8 \mathrm{~kb}$ is derived from the HMW-GS gene and that of $0.9 \mathrm{~kb}$ is derived from the LMW-GS gene.

analysis showed that these $\mathrm{F}_{1}$ plants harbored both HMWGS and LMW-GS genes (Fig. 5, lanes 1 and 2). Homozygous plants with both HMW-GS and LMW-GS genes were selected by PCR using primer sets specific for the HMW-GS and LMW-GS genes in the $\mathrm{F}_{3}$ generation, and these were designated as HLMW-GS as a line. In the transgenic rice lines, the estimation of the copy number of the GS gene by Southern analysis with probes specific for GSs was not carried out, because there were no appropriate restriction enzyme sites in the introduced large wheat DNA fragment to detect restriction fragments containing the neighboring rice genome sequence, which should vary among the copies. However, in the PCR analysis of the $\mathrm{F}_{2}$ generation from a cross between the HMW-113 and LMW-228 lines, plants with and without the GS gene were segregated in the ratio of $3: 1$ (HMW-GS; $35: 13, \chi^{2}=0.111, \mathrm{P}=0.74$, LMW-GS; $\left.33: 15, \chi^{2}=1.000, P=0.32\right)$. This result indicated that each HMW-GS and LMW-GS gene was integrated into the rice genome as a single locus in the transgenic rice lines.

\section{Expression of $H M W$ - and $L M W-G S$ in transgenic rice lines}

Fig. 6 shows the total protein composition in the transgenic rice lines. In the HMW-113 and LMW-228 lines, protein bands around 75 and $37 \mathrm{kDa}$, respectively, were identified by SDS-PAGE (lanes 2 and 3). In the HLMW-GS line, both protein bands were detected (lane 4), unlike in the LGC1. It was expected that these transgenic-specific protein bands corresponded to the GS proteins. We analyzed the expression of the wheat glutenin genes in the three transgenic lines by 2D-PAGE and determined the N-terminal amino acid sequence of the proteins. Fig. 7 shows the expression of wheat GSs in the transgenic rice seeds, as revealed by 2D-PAGE analysis. In the LGC1, neither HMW-GS nor LMW-GS was detected (Fig. 7A). In the HMW-113 and the LMW-228 lines, protein spots around 75 and $37 \mathrm{kDa}$, respectively, were identified by 2D-PAGE (Fig. 7B and C). In the HLMW-GS line, both protein spots were detected (Fig. 7D). The N-terminal amino acid sequences of the 75 and $37 \mathrm{kDa}$ proteins corresponded to the mature HMW-GS

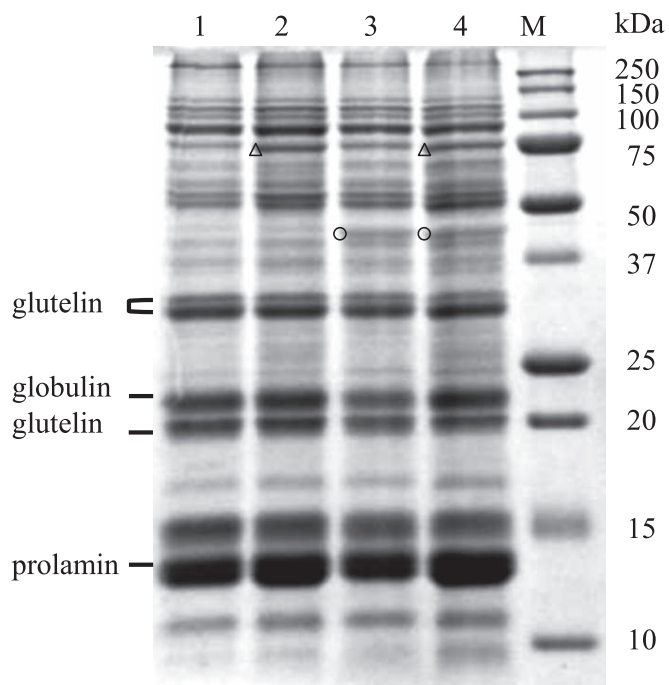

Fig. 6. Total protein composition of transgenic rice lines. 1, LGC1, 2, HMW-113, 3, LMW-228, and 4, HLMW-GS. Protein bands around 75 and $37 \mathrm{kDa}$ are indicated by white arrowheads and white circles, respectively.

(EGEASGQLQ) and LMW-GS (ISQQQQQ) proteins, which were processed at the same positions as those of the mature GS proteins within wheat seeds (Fig. 3).

HMW-GS in the HMW-113 line and LMW-GS in the LMW-228 line were extracted either as glutenin fraction with $50 \%$ of 1 -propanol or as total storage protein with $2 \%$ of SDS in the presence of 1\% DTT (Fig. 8A-D). In the LMW-228 and HLMW-GS lines, the amount of LMW-GS extracted with only $50 \%$ of 1-propanol (Fig. 9, lanes 3 and 4), was smaller than that of LMW-GS extracted with $50 \%$ of 1-propanol in the presence of 1\% DTT (Fig. 9, lanes 7 and 8).

\section{Discussion}

We isolated two TAC clones, 3-113 and 4-228, harboring a HMW-GS gene and a LMW-GS gene, respectively. Based on our results, we were able to characterize these GS genes in the selected clones. The expected $\mathrm{N}$-terminal amino acid sequence of HMW-GS of clone 3-113 was identical to that of B1y9 (Fig. 3A). The HMW-GS gene-specific primer detected the gene on chromosome $1 \mathrm{~B}$ in Chinese Spring (Fig. 4A). The Glu-B1 gene on chromosome 1B that encoded a y-type HMW-GS in Chinese Spring corresponded to that of B1y8 (Payne et al. 1980). Therefore, the HMW-GS gene of clone 3-113 corresponded to that of B1y8 encoded by the Glu-B1 gene on chromosome 1B in Chinese Spring. The expected N-terminal amino acid sequence of LMW-GS of clone 4-228 had showed a high similarity to that of Group 11 or Group 12 (Fig. 3B), and the LMW-GS gene-specific primer detected the gene on chromosome 1A in Chinese Spring (Fig. 4B). Therefore, the LMW-GS gene of clone 4-228 belonged to Group 11 encoded by the Glu-A1 gene on chromosome 1A in Chinese Spring (Ikeda et al. 2006).

Clones 3-113 and 4-228 also contained some motifs 

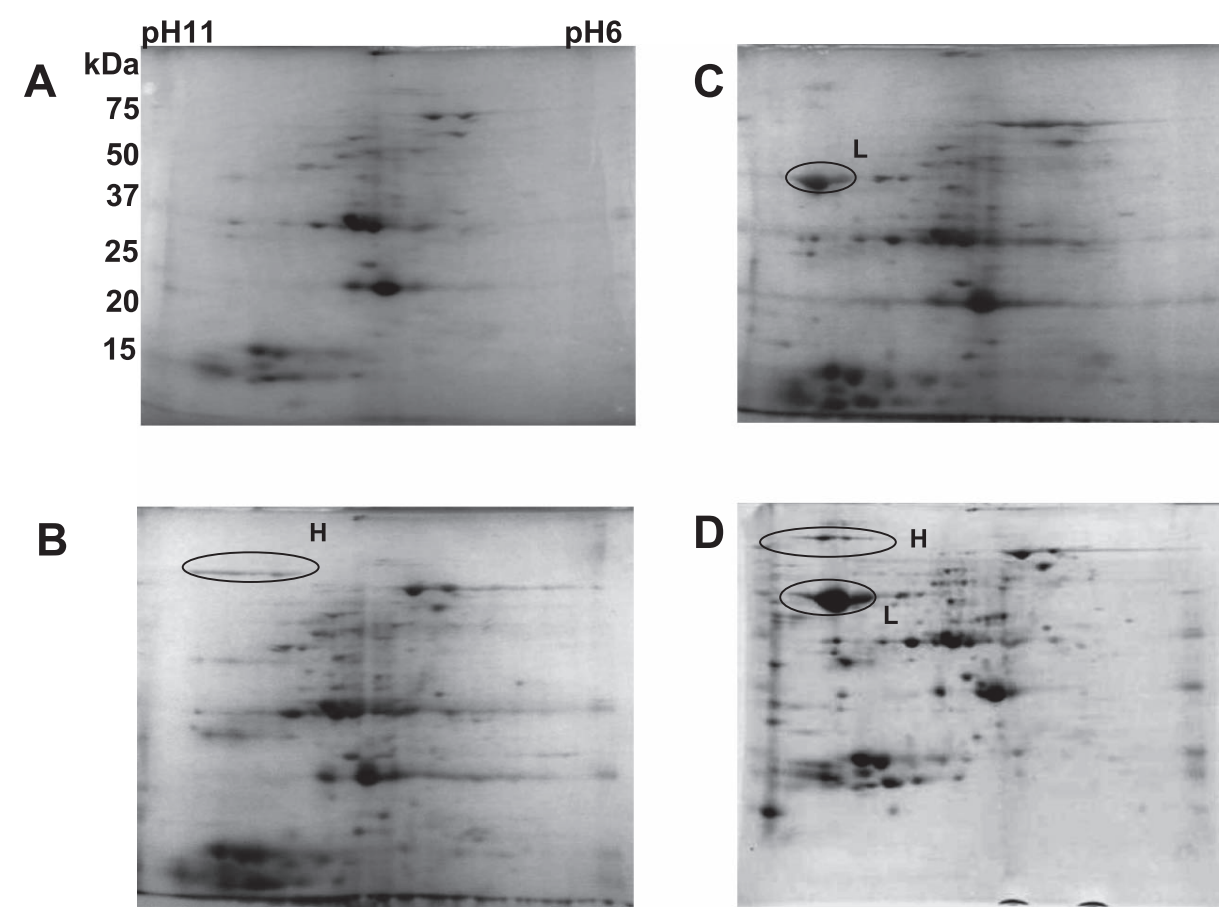

Fig. 7. 2D gel electrophoresis of total storage proteins in transgenic rice lines. (A) LGC1, (B) HMW-113, (C) LMW-228 and (D) HLMW-GS. The LMW-GS and HMW-GS proteins are indicated by the letters $\mathrm{L}$ and $\mathrm{H}$, respectively.
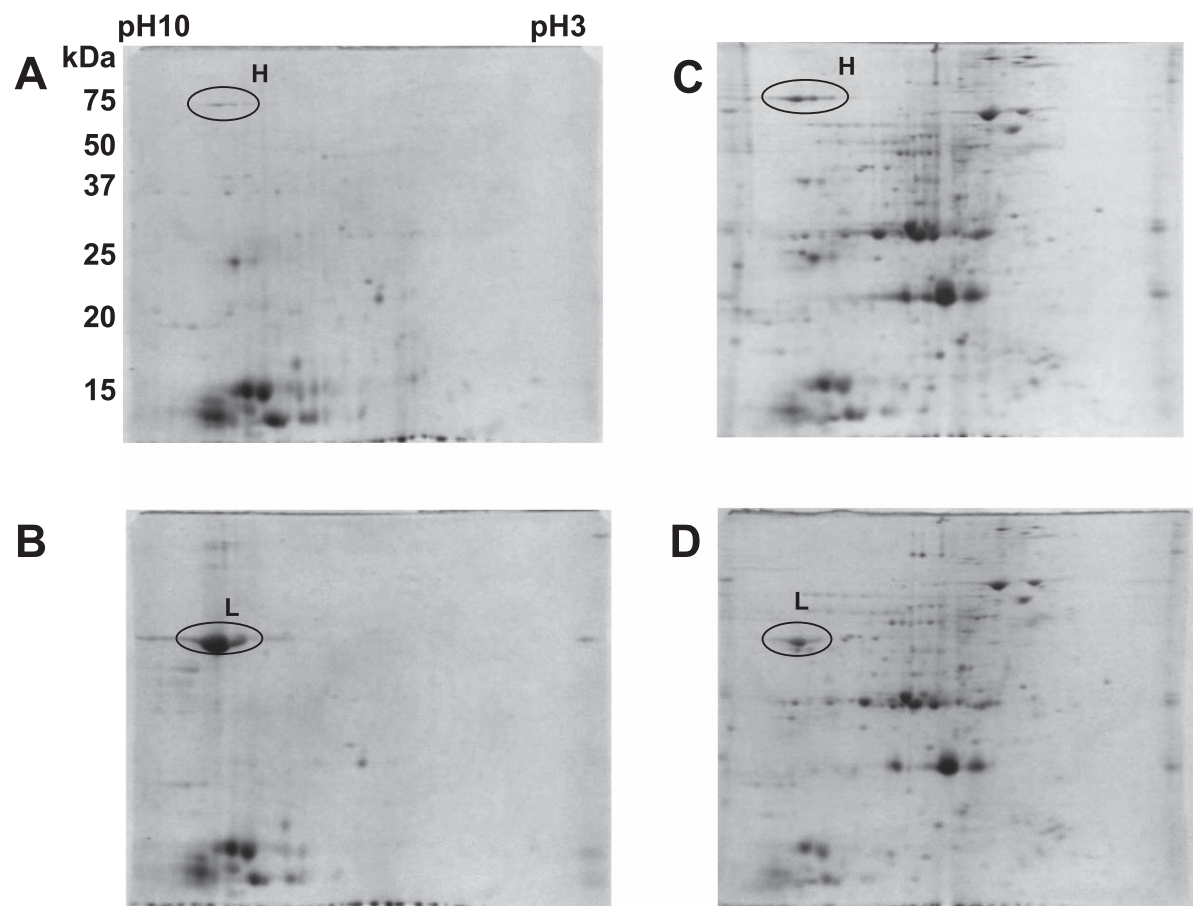

Fig. 8. 2D gel electrophoresis of glutenin fractions and total storage proteins in HMW-113 (A, C) and LMW-228 (B, D). A and B, glutenin fractions extracted with $50 \%$ of 1 -propanol in the presence of $1 \%$ DTT. C and D, total storage proteins extracted with $2 \%$ of SDS in the presence of $1 \%$ DTT. The LMW-GS and HMW-GS proteins are indicated by the letters L and H, respectively.

in the $5^{\prime}$ upstream region, which might be associated with endosperm-specific gene expression (Fig. 2). In clone 3-113, a HMW prolamin promoter (Thomas and Flavell 1990), two GCN4 boxes (Takaiwa et al. 1996) and an AACA motif (Takaiwa et al. 1996) were identified in the $5^{\prime}$ upstream region of the HMW-GS gene (Fig. 2A). Clone 4-228 contained LMW-GS, LMW prolamin boxes (Wu et al. 2000), an ACGT motif (Schmidt et al. 1992) and three GCN4 boxes (Takaiwa et al. 1996) in the $5^{\prime}$ upstream region of the LMW-GS gene (Fig. 2B). Wu et al. (2000) reported that the 


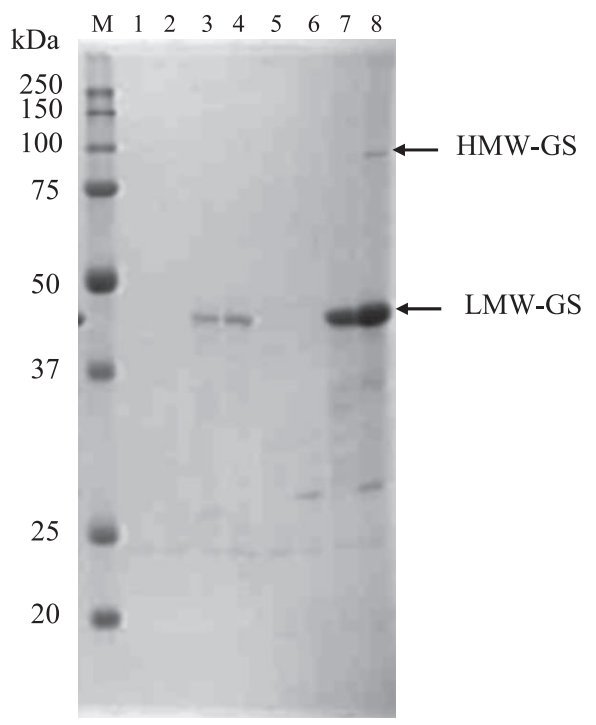

Fig. 9. SDS-PAGE analysis of GS in transgenic rice lines. 1 and 5, LGC1, 2 and 6, HMW-113, 3 and 7, LMW-228, 4 and 8, HLMW-GS. 1, 2, 3 and 4, 50\% 1-propanol-soluble fractions, 5, 6, 7 and 8, 50\% 1-propanol- and 1\% DTT-soluble fractions. The LMW-GS and HMW-GS proteins are indicated by the arrows.

GCN4 motif acted as an essential element determining the endosperm-specific expression and that AACA and ACGT and the prolamin box were involved in quantitative regulation of the endosperm-specific gene. Therefore, we expected that the selected clones contained cis-elements necessary for the expression of the GS genes in the wheat endosperm. Using these clones, we developed two lines of transgenic rice, namely HMW-113 harboring the gene coding for HMW-GS, and LMW-228 harboring the gene coding for LMW-GS. Moreover, by crossing these transgenic lines, a novel line, HLMW-GS harboring both genes was also developed. In the transgenic rice lines HMW-113 and LMW-228, the HMWGS and LMW-GS proteins accumulated within the endosperm, respectively (Fig. 7), confirming that these clones contained all the cis-elements for the proper expression of GS (Fig. 2), as expected from the complete sequences.

The expressed GS proteins in the transgenic lines were processed at the same site as that of the mature GS protein in wheat seeds, and accumulated as mature GS proteins within the transgenic rice seeds. This indicated that HMW-GS and LMW-GS were expressed within transgenic rice seeds, and were properly processed, as in the case of the wheat seeds. Disulfide bonding among HMW-GSs and LMW-GSs is important for the formation of a glutenin network with a large amount of polymeric GS proteins. In the transgenic rice line HLMW-GS, similar amounts of HMW-GS and LMW-GS to those in HMW-113 and LMW-228 accumulated, respectively (Fig. 7). These results suggested that HMW-GS and LMW-GS did not compete with each other for accumulation within rice seeds. This is the first study showing that the two kinds of wheat GSs, HMW-GS and LMW-GS, could accu- mulate in the rice endosperm.

These GS proteins were preferentially extracted by either $50 \%$ of 1-propanol or $2 \%$ of SDS in the presence of $1 \%$ DTT (Fig. 8). The results suggested that these proteins had formed insoluble polymeric proteins within the transgenic rice endosperm and that they may be located with prolamins in PB-I.

In the present study, we showed that wheat GSs were processed and accumulated within the rice endosperm, although wheat GSs accumulate as a continuous proteinaceous matrix surrounding the starch granules in wheat seeds (Parker 1982), in contrast to the two kinds of protein bodies for storage in rice seeds (Okita and Rogers 1996, Tanaka et al. 1980). In the transgenic rice lines expressing LMWGSs, a small amount of LMW-GS was found in the 50\% 1-propanol-soluble fraction, while most of LMW-GS was extracted in the insoluble fraction (Fig. 9), suggesting that most of the GSs expressed in the transgenic lines formed insoluble polymeric proteins. In the transgenic rice lines expressing soybean glycinins, unprocessed proglycinins had been detected in the endosperm (Katsube et al. 1999). The results obtained in the present study suggested that the proteinprocessing system was conserved between rice and wheat, and that it was possible to produce wheat gluten using the protein-processing system of rice.

\section{Literature Cited}

Fukuoka,H., T.Ogawa, I.Mitsuhara, T.Iwai, K.Isuzugawa, Y.Nishizawa, Y.Gotoh, Y.Nishizawa, A.Tagiri, M.Ugaki et al. (2000) Agrobacterium-mediated transformation of monocot and dicot plants using the NCR promoter derived from soybean chlorotic mottle virus. Plant Cell Rep. 19: 815-820.

Gupta, R.B. and K.W. Shepherd (1990) Two-step one-dimensional SDS-PAGE analysis of LMW subunits of glutelin. 1. Variation and genetic control of the subunits in hexaploid wheats. Theor. Appl. Genet. 80: 65-74.

Iida,S., E.Amano and T.Nishio (1993) A rice (Oryza sativa L.) mutant having a low content of glutelin and a high content of prolamine. Theor. Appl. Genet. 87: 374-378.

Iida, S., M.Kusaba and T.Nishio (1997) Mutants lacking glutelin subunits in rice: mapping and combination of maturated glutelin genes. Theor. Appl. Genet. 94: 177-183.

Ikeda,T.M., T.Nagamine, H.Fukuoka and H.Yano (2002) Characterization of new low-molecular-weight glutenin subunit genes in wheat. Theor. Appl. Genet. 104: 680-687.

Ikeda,T.M., N.Ohnishi, T.Nagamine, S.Oda, T.Hisamoto and H.Yano (2005) Identification of new puroindoline genotypes and their protein products among wheat cultivars. J. Cereal Sci. 41: 1-6.

Ikeda,T.M., E.Araki, Y.Fujita and H.Yano (2006) Characterization of low-molecular-weight glutenin subunit genes and their protein products in common wheats. Theor. Appl. Genet. 112: 327334.

Jackson, E., L.Holt and P.Payne (1983) Characterisation of highmolecular-weight gliadin and low-molecular-weight glutenin subunits of wheat endosperm by two-dimensional electrophoresis and the chromosomal location of their controlling genes. Theor. Appl. Genet. 66: 29-37. 
Katsube, T., N. Kurisaka， M. Ogawa， N. Maruyama， R. Ohtsuka, S.Utsumi and F.Takaiwa (1999) Accumulation of soybean glycinin and its assembly with the glutelins in rice. Plant Physiol. 120: 1063-1073.

Liu,Y.-G., K.Nagaki, M.Fujita, K.Kawaura, M.Uozumi and Y.Ogihara (2000) Development of an efficient maintenance and screening system for large-insert genomic DNA libraries of hexaploid wheat in a transformation-competent artificial chromosome (TAC) vector. Plant J. 23: 687-695.

Neuhoff, V., N. Arold, D. Taube and W.Ehrhardt (1988) Twodimensional fractionation of the endosperm proteins of bread wheat (Triticum aestivum L.): biochemical and genetic studies. Cereal Chem. 9: 255-262.

Ogawa,M., T.Kumamaru, H.Satoh, N.Iwata, T.Omura, Z.Kasai and K. Tanaka (1987) Purification of protein body-I of rice seeed and its polypeptide composition. Plant Cell Physiol. 28: 15171527.

Okita,T.W. and J.C.Rogers (1996) Compartmentation of proteins in the endomembrane system of plant cells. Annu. Rev. Plant Physiol. Plant Mol. Biol. 47: 327-350.

Parker,M.L. (1982) Protein accumulation in the developing endosperm of a high-protein line of Triticum dicoccoides. Plant Cell Environ. 5: 37-43.

Payne,P.I., C.N.Law and E.E.Mudd (1980) Control by homoeologous group 1 chromosomes of the high-molecular weight subunits of glutenin, a major protein of wheat endosperm. Theor. Appl.

\section{Note added to the proof}

Oszvald et al. reported the expression of another HMW-GS protein in transgenic rice in Cereal Research Communications, in December, 2007.

Oszvald,M., B.Jenes, S.Tomoskozi, F.Bekes and L.Tamas (2007) Expression of the 1Dx5 high molecular weight glutenin subunit protein in transgenic rice. Cereal Res. Commun. 35: 15431549 .
Genet. 58: 113-120.

Schmidt, R.J., M.Ketudat， M.J.Aukerman and G.Hoschek (1992) Opaque- 2 is a transcriptional activator that recognizes a specific target site in 22-kD zein genes. Plant Cell 4: 689-700.

Shewry,P.R., J.A.Napier and A.S.Tatham (1995) Seed storage proteins: structures and biosynthesis. Plant Cell 7: 945-956.

Takaiwa,F., U.Yamanouchi, T. Yoshihara, H.Washida, F. Tanabe, A.Kato and K.Yamada (1996) Characterization of common cis-regulatory elements responsible for the endosperm-specific expression of members of the rice glutelin multigene family. Plant Mol. Biol. 330: 1207-1221.

Tanaka,K., T.Sugimoto, M.Ogawa and Z.Kasai (1980) Isolation and characterization of two types of protein bodies in rice endosperm. Agric. Biol. Chem. 44: 1633-1639.

Thomas, M.S. and R.B.Flavell (1990) Identification of an enhancer element for the endosperm-specific expression of high molecular weight glutenin. Plant Cell 2: 1171-1180.

Wu,C.-Y., H.Washida, Y.Onodera, K.Harada and F.Takaiwa (2000) Quantitative nature of the prolamin-box, ACGT and AACA motifs in a rice glutelin gene promoter: minimal cis-element requirements for endosperm-specific gene expression. Plant Journal 23: 415-421.

Yamagata,H., T.Sugimoto, K.Tanaka and Z.Kasai (1982) Biosynthesis of storage proteins in developing rice seeds. Plant Physiol. 70: 1094-1100. 